\title{
Moments and mean square charge radii of short-lived argon isotopes
}

\author{
A. Klein ${ }^{1}$, B. A. Brown ${ }^{2}$, U. Georg ${ }^{1}$, M. Keim ${ }^{1}$, P. Lievens ${ }^{3}$, \\ R. Neugart ${ }^{1}$, M. Neuroth ${ }^{1}$, R. E. Silverans ${ }^{3}$, L. Vermeeren ${ }^{4}$ \\ and the ISOLDE Collaboration ${ }^{5}$ \\ ${ }^{1}$ Institut für Physik, Universität Mainz, \\ D-55099 Mainz, Germany \\ 2 National Superconducting Cyclotron Laboratory and \\ Department of Physics and Astronomy, Michigan State University, \\ East Lansing, MI 48824, USA \\ ${ }^{3}$ Laboratorium voor Vaste-Stoffysika en Magnetisme, K.U. Leuven, \\ B-3001 Leuven, Belgium \\ ${ }^{4}$ Instituut voor Kern- en Stralingsfysika, K.U. Leuven, \\ B-3001 Leuven, Belgium \\ ${ }^{5}$ CERN, CH-1211 Geneva 23, Switzerland
}

\begin{abstract}
We report on the measurement of optical isotope shifts for ${ }^{32-40} \mathrm{Ar}$ and for ${ }^{46} \mathrm{Ar}$ from which the changes in mean square nuclear charge radii across the $N=20$ neutron shell closure are deduced. The investigations were carried out by collinear laser spectroscopy in fast beams of neutral argon atoms. The ultra-sensitive detection combines optical pumping, stateselective collisional ionization and counting of $\beta$-radioactivity. By reaching far into the sd-shell, the results add new information to the systematics of radii in the calcium region $(Z \approx 20)$. Contrary to all major neutron shell closures with $N \geq 28$, the $N=20$ shell closure causes no significant slope change in the development of the radii. Information from the hyperfine structure of the odd- $A$ isotopes includes the magnetic moments of ${ }^{33} \mathrm{Ar}(I=1 / 2)$ and ${ }^{39} \mathrm{Ar}(I=7 / 2)$, and the quadrupole moments of ${ }^{35} \mathrm{Ar},{ }^{37} \mathrm{Ar}(I=3 / 2)$ and ${ }^{39} \mathrm{Ar}$. The electromagnetic moments are compared to shell-model predictions for the sd- and fp-shell. Even far from stability a very good agreement between experiment and theory is found for these quantities. The mean square charge radii are discussed in the framework of spherical SGII Skyrme-type Hartree-Fock calculations.
\end{abstract}

Keywords: NUCLEAR STRUCTURE ${ }^{32-40,46} \mathrm{Ar}$; measured isotope shifts, hfs; deduced hyperfine constants, spins $I$, magnetic dipole moments $\mu$, electric quadrupole moments $Q_{s}$, mean square charge radii $\left\langle r^{2}\right\rangle$; Collinear fast-beam laser spectroscopy, collisional ionization and $\beta$-detection; shell model (sd-, fp-shell). 


\section{Introduction}

The systematics of the mean square charge radii of nuclei in the calcium region is determined by several proton and neutron shell closures. Measurements on the six stable calcium isotopes $(Z=20)$ cover the $1 \mathrm{f}_{7 / 2}$-shell between the doubly magic nuclei ${ }^{40} \mathrm{Ca}$ $(N=20)$ and ${ }^{48} \mathrm{Ca}(N=28)$. The charge radii of these calcium nuclei have been studied by muonic X-ray spectroscopy [1, 2], by electron scattering [3] as well as by optical isotope shift measurements $[4,5]$. Several theoretical attempts towards a quantitative interpretation of these data have been made $[6,7,8,9,10]$. In the vicinity of this region the information about radii is rather scarce. Isotope shift measurements on ${ }^{38-47} \mathrm{~K}[11]$ just include one nucleus of the sd-shell $(Z, N<20)$. The corresponding ${ }^{39} \mathrm{Ca}$ has been investigated recently [12], and a similar collinear laser spectroscopy experiment on ${ }^{50} \mathrm{Ca}$ has extended the calcium data into the $2 \mathrm{p}_{3 / 2}$-subshell beyond $N=28$ [13]. With the present investigation of argon $(Z=18)$ isotopes with neutron numbers between $N=14$ and $N=28$ we contribute to a more global systematics of the charge radii in the region around the $N, Z=20$ and the $N=28$ shell closures.

Another solid piece of information is obtained from the measurement of hyperfine structures of the odd- $A$ isotopes. Magnetic moments and electric quadrupole moments of nuclear ground states (far from stability) can be derived from these. The nuclear moments, being experimentally and theoretically well defined properties, provide a test for any quantitative nuclear theory. For nuclei with $A=17-39$, the shell-model calculations of Brown and Wildenthal [14] using the full sd-shell $\left(2 \mathrm{~s}_{1 / 2}, 1 \mathrm{~d}_{5 / 2}, 1 \mathrm{~d}_{3 / 2}\right)$ as the active model space together with the W (USD) interaction [15] have been remarkably successful in predicting a large variety of nuclear properties. One of the disagreements of these theoretical sd-shell calculations with experiment is for the Gamow-Teller $\beta$-decay strength in the upper part of the sd-shell, in particular as measured in the decay of the neutrondeficient $\mathrm{Ar}$ [16] and $\mathrm{Ca}$ [17] isotopes. Theoretically there appears to be too little strength at medium excitation energy, which is probably related to the centroid of the GamowTeller resonance being predicted several MeV too high compared to experiment [18]. However, the predictions of the $\mathrm{W}$-interaction for the Gamow-Teller decay to low-lying states $[17,19]$, as well as for the ground state moments which we will discuss in this paper, are in good agreement with experiment. The Gamow-Teller matrix element is related to the isovector part of the magnetic moment by isospin invariance. Thus the determination of the magnetic moment of ${ }^{33} \mathrm{Ar}$ is highly desirable for a check of the validity of the shell-model calculations far from stability.

Optical investigations of radii and moments of nuclei with $Z<20$ require particularly high experimental resolution because of the atomic properties of light elements. The isotope shift is a superposition of two contributions, namely the field shift and the mass shift. For light elements, the field shifts from which the changes in mean square charge radii are derived, appear as a small effect. This is a consequence of the low electron density probing the charge distribution of the nuclei. Moreover, the isotope shift is dominated by the mass shift arising from the change in the recoil energy of the nucleus as a function of the mass. Despite these peculiarities the main experimental challenge of such optical investigations far from stability is the extreme sensitivity required due to the 
low production yield coming along with very unfavourable background conditions.

These experimental requirements are met by the method of collinear fast-beam laser spectroscopy combined with a selective and ultra-sensitive resonance detection technique. This technique based on optical pumping, state-selective collisional ionization and observation of the $\beta$-decay of collected ions is described in Section 2.1. The specific effort that was made to calibrate the large Doppler shifts encountered with beams of light nuclides is discussed in Section 2.2. The results are presented in Section 3, and the measured nuclear moments and radii are discussed in Section 4.

\section{Experiment}

\subsection{Ultra-sensitive laser spectroscopy on argon}

The hyperfine structure and isotope shift of ${ }^{32-40} \mathrm{Ar}$ and ${ }^{46} \mathrm{Ar}$ in the transition $4 \mathrm{~s}[3 / 2]_{2} \rightarrow$ $4 \mathrm{p}[3 / 2]_{2}(\lambda=764 \mathrm{~nm})$ of the atomic spectrum of argon were measured by collinear laser spectroscopy [20]. The experiments were carried out on-line at the new ISOLDE mass separator at the CERN PS-Booster synchrotron [21].

The radioactive argon atoms were formed in the $\mathrm{CaO}$ target of ISOLDE by spallation reactions of the type ${ }^{A} \mathrm{Ca}(\mathrm{p}, 3 \mathrm{p} X \mathrm{n}){ }^{A-2-X} \mathrm{Ar}$. These reactions were induced by the incidence of $1 \mathrm{GeV}$ protons from the PS-Booster. Proton pulses containing typically $2 \times 10^{13}$ protons with a pulse length of $2.4 \mu$ s are impinging on the target approximately every $2.4 \mathrm{~s}$. Since the target material was of natural isotopic abundance $\left(97 \%\right.$ of $\left.{ }^{40} \mathrm{Ca}\right)$, the production of neutron deficient argon isotopes with $A \leq 38$ was strongly favoured.

${ }^{46} \mathrm{Ar}$ (produced from ${ }^{48} \mathrm{Ca}$ ) was the heaviest argon isotope accessible. A cooled transfer line connected the target to a plasma ion source from which the ions were extracted and accelerated electrostatically to $60 \mathrm{keV}$. Although the cold transfer line suppresses the contamination of noble gas beams by less volatile isobars, huge amounts of stable isobaric gaseous molecules present all over the mass region enter the ionization volume. These isobaric contaminations are not separated from the isobaric radioactive ion beams by the GPS (general purpose separator) magnet of ISOLDE which has a resolving power of $m / \Delta m=2400$. For instance, the beam of mass $m=32$ a.m.u. contained less than $10^{3}$ short-lived ${ }^{32} \mathrm{Ar}$ ions per second on a background exceeding $10^{8}$ ions per second.

The experimental setup is shown schematically in Figure 1. The mass-separated ion beam is superimposed collinearly on a $\mathrm{cw}$ laser beam. The ions are then neutralized by charge exchange in a potassium vapour cell. This charge exchange reaction favours the population of the $5 \mathrm{p}^{5} 4 \mathrm{~s}$ states in atomic argon at a binding energy of $4.2 \mathrm{eV}$ because of the quasi-resonant electron transfer from a potassium atom which has an ionization energy of $4.3 \mathrm{eV}$ (see Figure 2). Assuming a population distribution according to the statistical weights, roughly $40 \%$ of the argon atoms should be formed in the metastable $4 \mathrm{~s}[3 / 2]_{2}$ state. Atoms in this state can be resonantly excited by the laser light to the $4 \mathrm{p}[3 / 2]_{2}$ state which decays either back to $4 \mathrm{~s}[3 / 2]_{2}$ or cascades via $4 \mathrm{~s}[3 / 2]_{1}$ or $4 \mathrm{~s}[1 / 2]_{1}$ to the $3 \mathrm{p}^{6}{ }^{1} \mathrm{~S}_{0}$ ground state (see Figure 2).

An electrostatic variation of the beam energy by a computer-controlled electrical potential applied to the charge exchange cell is used to tune the optical frequency in the rest frame of the atoms. As the total Doppler shift is about $700 \mathrm{GHz}$, a few hundred volts are sufficient to cover all hyperfine structures and isotope shifts (see results), but 
isotope shift measurements over up to 8 mass numbers require $\pm 7 \mathrm{keV}$ changes of the beam energy because of the large differences in the Doppler shifts (see section 3.2). The optical resonance signals can be detected by counting the fluorescence photons from the decay of the $4 \mathrm{p}[3 / 2]_{2}$ state. This traditional method has been applied for argon beams with intensities exceeding $10^{6}$ ions/s. A drawback of the optical detection scheme is the low counting efficiency for fluorescence photons of about $10^{-4}$, arising from the solid angle of the collection optics and the quantum efficiency of the photomultiplier. Moreover, a constant background from stray laser light is inherent to this technique.

The much more efficient collisional ionization detection [22] takes advantage of the state dependence of the cross-sections for electron stripping in atomic collisions. Downstream of the optical excitation region the atomic beam passes through a $\mathrm{Cl}_{2}$ gas target. Here the neutral argon atoms are reionized in collisions with the $\mathrm{Cl}_{2}$ molecules. The created ions are separated from the remaining atomic beam in an electrostatic deflector and counted by secondary electron detection. For the argon atoms in the loosely bound high-lying metastable $4 \mathrm{~s}[3 / 2]_{2}$ state, the ionization cross-section is about 3 times larger than for those in the ground state. At resonance in the transition $4 \mathrm{~s}[3 / 2]_{2} \rightarrow 4 \mathrm{p}[3 / 2]_{2}$, the metastable state is depopulated into the ground state by optical pumping. This leads to an appreciable drop in the ion count rate.

The pressure of the target gas was optimized during an off-line test on stable ${ }^{36} \mathrm{Ar}$, in order to obtain an optimum signal-to-noise ratio for the resonance detection. Off resonance, the argon atoms were ionized with an efficiency of $\varepsilon_{i o n}=0.09$ at a pressure of $1.3 \times 10^{-1} \mathrm{~Pa}$ equivalent to a target density of $3.5 \times 10^{14}$ molecules per $\mathrm{cm}^{2}$. The relative signal depth for the resonance in ${ }^{36} \operatorname{Ar}(I=0)$ was $S=0.25$. For isotopes with spin $I \neq 0$, this signal strength is distributed over several hyperfine structure components.

Although this technique provides a detection efficiency superior to the fluorescence photon counting and does not suffer from background due to the stray laser light, its application is restricted to rather pure beams. It has been pointed out that this condition is by far not fulfilled in the argon mass region. The purification of the contaminated beams by the specific neutralization and collisional ionization processes turns out to be rather poor. Hence an isotope-selective detection of the argon ions is the conditio sine qua non of the resonance detection for isotopes far from stability.

For short-lived isotopes, this selectivity is achieved by detecting the radioactivity. The reionized fraction of the beam is guided into a $\beta$-ray detection system consisting of a tape transport and a scintillation counter (see Figure 1). The detector consists of two plastic scintillator plates (NE102, $1 \mathrm{~mm}$ thickness, $30 \mathrm{~cm}^{2}$ surface) mounted on a light guide with a photomultiplier readout. A $14 \mathrm{~mm}$ wide tape is placed between these two plates. The ion beam is implanted into the tape through a hole in the front scintillator. The $\beta$-activity of the implanted nuclei is detected in situ with a solid angle of about $70 \%$ of $4 \pi$.

The total efficiency for the detection of short-lived argon ions produced by the ISOLDE separator is approximately 2-3\%. Apart from the limited efficiency for the collisional reionization of argon atoms, transmission losses, incomplete neutralization and the solid angle of the $\beta$-detection determine this number.

After each measuring interval corresponding to one channel of the spectrum, longlived activity is transported out of the detection region by moving the tape via a stepping motor drive, and the optical excitation frequency is changed by setting an appropriate retardation voltage for the next channel. The implantation and the $\beta$-counting is triggered 
by the impact of the PS-Booster pulse on the ISOLDE target. The duration of the implantation and $\beta$-counting is adapted to the specific half-life and background conditions found for a certain isotope. For instance, in case of ${ }^{32} \mathrm{Ar}\left(T_{1 / 2}=98 \mathrm{~ms}\right)$ the implantation was stopped $300 \mathrm{~ms}$ after the proton impact, while the $\beta$-decay was observed for $500 \mathrm{~ms}$. This ensures a reduction of isobaric background activity from $\mathrm{N}_{18} \mathrm{~N}_{14}$ molecules [23] $\left(T_{1 / 2}\right.$ $=630 \mathrm{~ms}$ ) to a level of about $35 \%$ (see Figure $3 \mathrm{a}$ ). An optical resonance on ${ }^{32} \mathrm{Ar}$ could be detected with a signal-to-noise ratio of 7 within a total integration time of 6 min per channel (see Figure $3 \mathrm{~b}$ ). In this ultimate case the ${ }^{32} \mathrm{Ar}$ yield from the separator was about 300 ions per second which is close to the sensitivity limit of the technique.

The applicability of the radioactivity detection scheme obviously depends on the half-life of the isotope under investigation. The $\beta$-detection technique was applied in measurements on ${ }^{32-35} \operatorname{Ar}\left(T_{1 / 2}=0.1-1.8 \mathrm{~s}\right)$ and on ${ }^{46} \operatorname{Ar}\left(T_{1 / 2}=7.8 \mathrm{~s}\right)$. For isotopes with longer half-lives the sensitivity is considerably reduced. In order to avoid the influence of long-term instabilities or laser frequency drifts during an individual scan of the spectrum, the scanning has to be fast enough $(<10 \mathrm{~s} /$ channel $)$, which involves the incomplete observation of a slow $\beta$-decay. Already for half-lives of a few minutes the efficiency of the radioactivity detection decreases to $10^{-4}-10^{-3}$. This is comparable to the conditions obtained with the standard fluorescence detection scheme. Consequently, the fluorescence detection was used for measurements on the long-lived or stable isotopes ${ }^{37-40} \mathrm{Ar}$.

\subsection{Data taking and frequency calibration}

Before and after the measurement on a radioactive isotope, resonances of two stable reference isotopes, ${ }^{38} \mathrm{Ar}$ and ${ }^{36} \mathrm{Ar}$ or ${ }^{40} \mathrm{Ar}$, were recorded using the fluorescence detection scheme. The voltage $U$ corresponding to the resonance of ${ }^{38} \mathrm{Ar}$ serves as a reference for the determination of frequency differences $(\Delta \nu)$ arising from the hyperfine structure or the isotope shift in the rest frame of the atoms. The resonance of the second reference isotope as well as changes in the ${ }^{38} \mathrm{Ar}$ resonance position provide an on-line test for the stability of the laser frequency and the high voltage. At constant laser frequency $\nu_{0}$ the frequency difference $\Delta \nu$ is determined from the change in beam energy $(\sim \Delta U)$ required to tune the Doppler shifted laser frequency to the resonance of an isotope of mass $m+\Delta m$ with respect to the reference voltage at mass $m=38$ a.m.u. To first order it is given by

$$
\Delta \nu \approx \nu_{0}\left(\frac{\Delta m}{m}-\frac{\Delta U}{U}\right) \sqrt{\frac{e U}{2 m c^{2}}} .
$$

Thus the accuracy of the frequency calibration is limited by the accuracy of the highvoltage dividers employed for the measurement of the acceleration and the post-acceleration voltages. An accuracy of $10^{-4}$ is specified for both these dividers (Ross Engineering Corp. VD75-10-BDL-PB-M/F and Julie Research kV-10R). However, the correct extraction potential from the plasma ion source is not accessible to a direct measurement. From indirect information about the shifts produced by changing the ion source parameters one can conclude that it is close to the anode potential of the source. This problem would be solved by measuring accurately the large Doppler shift $\left(\Delta \nu_{\text {Doppler }} \approx \nu_{0} \beta_{m} \approx 700\right.$ $\mathrm{GHz}$ ) of the resonance absorption frequency of stable ${ }^{40} \mathrm{Ar}$ in the fast beam. As such a measurement would involve a complicated absolute wavelength calibration, we chose an alternative which is based on the compensation of the differences in the Doppler shifts 
and in the resonance frequencies for the stable rubidium isotopes ${ }^{85,87} \mathrm{Rb}$. This calibration measurement is performed by collinear laser spectroscopy in the atomic transition $5 \mathrm{~s}{ }^{2} \mathrm{~S}_{1 / 2} \rightarrow 5 \mathrm{p}^{2} \mathrm{P}_{3 / 2}$ [24]. If the atomic resonances occur at about the same beam energy $(\Delta U=0)$, it follows from eq. (1) that the difference between the Doppler shifts of the two isotopes (first term of the right-hand side), and thus the acceleration voltage $U$, is exclusively determined by the atomic masses and the frequency difference in the rest frame $(\Delta \nu)$. In the case of ${ }^{85,87} \mathrm{Rb}$ the well known hyperfine structure and isotope shift $[25,26]$ compensates the Doppler shift difference within a beam energy interval of less than 60 $\mathrm{eV}$ for a particular group of three hyperfine structure components of each isotope (see Figure 4). The relative position of these six resonances provides an independent measurement of the total acceleration voltage. The calibration of the post-acceleration voltage is verified by the additional information from the positions of the remaining hyperfine structure components. This procedure proofs that the systematic errors of the voltage measurements are of the order of, but never larger than the specified accuracy of $10^{-4}$ which therefore will be used for the evaluation of the isotope shifts.

\section{Results}

\subsection{Hyperfine structure and electromagnetic moments}

For the odd- $A$ argon isotopes the magnetic dipole and the electric quadrupole hyperfine interaction constants $A$ and $B$ were determined from the observed hyperfine splittings of both states, $4 \mathrm{~s}[3 / 2]_{2}$ and $4 \mathrm{p}[3 / 2]_{2}$, using a least square fitting procedure. They are compiled in Table 1 and Table 2. Earlier hyperfine structure data for ${ }^{37} \mathrm{Ar}$ and ${ }^{39} \mathrm{Ar}$ obtained from Doppler limited optical spectroscopy [27, 28] are much less accurate than the present results.

Values of the magnetic moments were deduced from the $A$-factors of the $4 \mathrm{~s}[3 / 2]_{2}$ state with reference to the NMR measurement on ${ }^{37} \mathrm{Ar}$ performed by Pitt et al. [29], according to the relation

$$
\frac{\mu_{I}^{x} I^{37}}{\mu_{I}^{37} I^{x}}\left(1-\Delta^{x, 37}\right)=\frac{A^{x}}{A^{37}} .
$$

The magnetic moment of ${ }^{37} \mathrm{Ar}$ was preferred as a reference to the more precisely known moment of ${ }^{35} \mathrm{Ar}$ [30], since the $A$-factor of the former isotope was determined more accurately in the present experiment. A calculation of the hyperfine anomaly $\Delta^{x, 37}$ based on the theoretical decomposition of the magnetic moments into spin, orbit and polarization contribution compiled in Table 4 (see Section 4.1.1) predicts negligible corrections of $\Delta^{x, 37} \approx 1 \times 10^{-4}$ for all cases [31,32]. The moments derived from relation $(2)$ and corrected for diamagnetic shielding [33] are included in Table 1.

The spectroscopic quadrupole moments $Q_{s}$ were calculated from the $B$-factors of the $4 \mathrm{~s}[3 / 2]_{2}$ level following the procedure outlined in [34] for the corresponding level in krypton. The electric field gradient is derived from the magnetic hyperfine structure constant $a_{p 3 / 2}=419 \mathrm{MHz}$ of the $\mathrm{p}_{3 / 2}$ hole according to a Breit-Wills analysis performed for the $4 \mathrm{p}^{5} 5 \mathrm{~s}$ configuration [24]. This yields the calibration factor

$$
B\left(4 \mathrm{~s}[3 / 2]_{2}\right) / Q_{s}=-1.01 \mathrm{MHz} / \mathrm{mb}
$$

with an uncertainty of approximately $10 \%$ including the estimated error of $a_{p 3 / 2}$ and an additional uncertainty covering the possible range of a Sternheimer correction. Since no 
Table 1: $A$-factors and magnetic moments of odd- $A$ argon isotopes. The magnetic moments are obtained with reference to the value for ${ }^{37} \mathrm{Ar}$ and corrected for diamagnetism [33]. Hyperfine anomaly corrections are negligible compared to the errors of the magnetic moments given by the statistical errors of the $A$-factors and the error of the reference moment.

\begin{tabular}{|c|c|c|c|c|c|}
\hline & $I$ & $\begin{array}{c}A\left(4 \mathrm{~s}[3 / 2]_{2}\right) \\
{[\mathrm{MHz}]}\end{array}$ & $\begin{array}{c}A\left(4 \mathrm{p}[3 / 2]_{2}\right) \\
{[\mathrm{MHz}]}\end{array}$ & $\begin{array}{c}\mu \\
{\left[\mu_{N}\right]}\end{array}$ & $\begin{array}{c}\mu^{\text {lit }} \\
{\left[\mu_{N}\right]}\end{array}$ \\
\hline${ }^{33} \mathrm{Ar}$ & $1 / 2$ & $-912.3(4.0)^{*)}$ & - & $-0.723(6)$ & - \\
${ }^{35} \mathrm{Ar}$ & $3 / 2$ & $266.0(1.8)$ & $149.6(1.5)$ & $0.633(7)$ & $0.633(2)^{a)}$ \\
${ }^{37} \mathrm{Ar}$ & $3 / 2$ & $482.1(0.3)$ & $271.6(0.4)$ & reference & $1.145(5)^{b)}$ \\
${ }^{39} \mathrm{Ar}$ & $7 / 2$ & $-286.1(1.4)^{*)}$ & - & $-1.588(15)$ & $-1.6(3)^{c)}$ \\
\hline
\end{tabular}

*) Analysis using $A\left(4 \mathrm{p}[3 / 2]_{2}\right) / A\left(4 \mathrm{~s}[3 / 2]_{2}\right)=0.5634(9)$ from ${ }^{37} \mathrm{Ar}$.

${ }^{a)}$ ref. [30]; ${ }^{b)}$ ref. [29]; ${ }^{c)}$ ref. [28].

value for the Sternheimer shielding factor has been published for argon, the correction is neglected following the arguments of the corresponding analyses for krypton [34] and xenon [35]. It should be pointed out that the ratios of quadrupole moments obtained from relation (3) are free from these systematic uncertainties. The final results for the spectroscopic quadrupole moments are included in Table 2.

Table 2: $B$-factors and electric quadrupole moments of odd- $A$ argon isotopes with $I>1 / 2$. The additional error of $10 \%$ applied to the quadrupole moments (in square brackets) accounts for the uncertainties in the evaluation of the electric field gradient and should include the missing Sternheimer shielding correction.

\begin{tabular}{|c|c|c|c|c|}
\hline & $I$ & $\begin{array}{c}B\left(4 \mathrm{~s}[3 / 2]_{2}\right) \\
{[\mathrm{MHz}]}\end{array}$ & $\begin{array}{c}B\left(4 \mathrm{p}[3 / 2]_{2}\right) \\
{[\mathrm{MHz}]}\end{array}$ & $\begin{array}{c}Q_{s} \\
{[\mathrm{mb}]}\end{array}$ \\
\hline${ }^{35} \mathrm{Ar}$ & $3 / 2$ & $84.8(7.2)$ & $45.2(5.2)$ & $-84(7)[8]$ \\
${ }^{37} \mathrm{Ar}$ & $3 / 2$ & $-77.0(1.6)$ & $-41.1(1.6)$ & $76.2(1.6)[7.6]$ \\
${ }^{39} \mathrm{Ar}$ & $7 / 2$ & $118(20)^{*)}$ & - & $-117(20)[12]$ \\
\hline
\end{tabular}

*) Analysis using $B\left(4 \mathrm{p}[3 / 2]_{2}\right) / B\left(4 \mathrm{~s}[3 / 2]_{2}\right)=0.535(24)$ from ${ }^{37} \mathrm{Ar}$.

\subsection{Isotope shifts and nuclear mean square charge radii}

Isotope shifts in the transition $4 \mathrm{~s}[3 / 2]_{2} \rightarrow 4 \mathrm{p}[3 / 2]_{2}$ were measured for ${ }^{32-40} \mathrm{Ar}$ and ${ }^{46} \mathrm{Ar}$. They are compiled in Table 3, where the errors in parentheses are purely statistical and the ones in square brackets are systematic errors, arising here from the voltage calibration. This error notation will be followed throughout the paper. The statistical errors arise from the determination of the resonance position (even- $A$ isotopes) or the position of the center of gravity (odd- $A$ isotopes) with respect to the reference position. They are mainly due to counting statistics and contain a small digitizing error of the post-acceleration voltage readout.

For light elements the isotope shift is small compared to the difference in the Doppler shifts, and thus the voltage difference between the resonances of two isotopes at constant 
laser frequency essentially compensates the Doppler shift difference. This means that

$$
\Delta U / U \approx \Delta m / m
$$

holds in good approximation. The isotope shift appears as a small effect on top of this large difference, and it has to be calculated from the voltages and masses using the relativistically correct formulas for the Doppler shift. It turns out that the relative errors of the high-voltage measurements enter here with the factor $\Delta U / U$ multiplied with the absolute Doppler shift. This means the large Doppler shift difference determines the error of the much smaller isotope shift. We shall see below that this systematic error can be eliminated in the procedure of evaluating the differences of mean square nuclear charge radii.

Starting from eq. (1) the systematic error of the isotope shift $\left(\epsilon\left(\delta \nu^{38, A}\right)\right)$ can be expressed by two parameters, $\kappa_{U}$ and $\kappa_{\Delta U}$, accounting for the uncertainties of the acceleration and the post-acceleration voltage calibrations:

$$
\epsilon\left(\delta \nu^{38, A}\right) \approx\left(\kappa_{U}+\kappa_{\Delta U}\right) \frac{m_{A}-m_{38}}{m_{A} m_{38}} .
$$

Here, for the sake of convenience, the mass term $\Delta m / m$ has been written in the form appearing in the expression for the mass shift (see eq. (7)). Assuming a calibration uncertainty of $1 \times 10^{-4}$ for the acceleration and the post-acceleration voltages the values of the constants $\kappa_{U}$ and $\kappa_{\Delta U}$ were determined to be

$$
\left|\kappa_{U}\right|=1324(2) \text { MHz a.m.u. ; }\left|\kappa_{\Delta U}\right|=1319(1) \text { MHz a.m.u. . }
$$

Both parameters were obtained by calculating the isotope shifts from the experimental data and varying the measured voltages independently within their error limits. Within the standard deviations (given in parentheses) $\kappa_{U}$ and $\kappa_{\Delta U}$ are constant over the whole chain of isotopes. The systematic errors of the isotope shifts in Table 3 are based on these values.

The isotope shift $\left(\delta \nu^{38, A}=\nu^{A}-\nu^{38}\right)$ can be decomposed into the field shift $\delta \nu_{f}^{38, A}$ and two terms accounting for the finite mass of the nucleus, the normal and the specific mass shift $\delta \nu_{n}^{38, A}$ and $\delta \nu_{s}^{38, A}$ :

$$
\delta \nu^{38, A}=\delta \nu_{f}^{38, A}+\delta \nu_{n}^{38, A}+\delta \nu_{s}^{38, A}=\delta \nu_{f}^{38, A}+\frac{m_{A}-m_{38}}{m_{A} m_{38}}\left(M_{n}+M_{s}\right) .
$$

The normal mass shift constant is given by $M_{n}=\nu m_{e}$ with the transition frequency $\nu$ and the electron mass $m_{e}$. The specific mass shift constant $M_{s}$ accounts for correlations of the electron motion. It is notoriously difficult to calculate, and in case of argon there is no prediction for $M_{s}$ available. Therefore the separation according to eq. (7) has been based on independent information about the field shifts $\delta \nu_{f}^{38,36}$ and $\delta \nu_{f}^{38,40}$ which is given by nuclear radii extracted from muonic X-ray spectroscopy data [2] for the stable isotopes.

Taking advantage of the similar mass dependencies of both the mass effect and the systematic (voltage) error of the isotope shift, the voltage calibration uncertainty can be eliminated in the evaluation of the field effect of the radioactive isotopes. By adding a correction of the form of eq. (5) to eq. (7) accounting for the possible miscalibration of the voltage, one obtains the experimentally determined isotope shift

$$
\delta \nu_{\text {exp }}^{38, A}=\delta \nu_{f}^{38, A}+\frac{m_{A}-m_{38}}{m_{A} m_{38}}\left(M_{n}+M_{s}+k_{U}+k_{\Delta U}\right) .
$$


Here $k_{u}$ and $k_{\Delta U}$ are unknown constants whose maximum absolute values should be of the order of $\kappa_{U}$ and $\kappa_{\Delta U}$ as assigned in eq. (6). They disappear if the voltage calibration assumed for the evaluation of isotope shifts is exactly correct. Multiplying eq. (8) with the inverse of the relative mass difference one obtains

$$
\delta \nu_{\text {exp }}^{38, A} \frac{m_{A} m_{38}}{m_{A}-m_{38}}=\delta \nu_{f}^{38, A} \frac{m_{A} m_{38}}{m_{A}-m_{38}}+M_{n}+C .
$$

The constant $C$ now contains the specific mass shift and a contribution due to the uncertainty of the voltages.

The field shift is related to the change in electron density at the site of the nucleus occurring in the optical transition, $\Delta|\Psi(0)|^{2}$, the function $f(Z)$ being sensitive to the course of the Dirac wave function at the site of the nucleus [36] and a nuclear parameter $\lambda^{38, A}$ which is - due to the low $Z$ - practically identical with the change in mean square charge radius $\delta\left\langle r^{2}\right\rangle^{38, A}[37]$,

$$
\delta \nu_{f}^{38, A}=F \lambda^{38, A} \approx F \delta\left\langle r^{2}\right\rangle^{38, A}=\pi a_{0}^{3} \frac{\Delta|\Psi(0)|^{2}}{Z} f(Z) \delta\left\langle r^{2}\right\rangle^{38, A} .
$$

Two values for the changes in nuclear mean square charge radii $\delta\left\langle r^{2}\right\rangle^{38,36}=-0.082 \mathrm{fm}^{2}$ and $\delta\left\langle r^{2}\right\rangle^{38,40}=0.171 \mathrm{fm}^{2}$ are obtained from the model dependent nuclear charge radii compiled by Fricke [2]. For both values a systematic error of $10 \%$ accounting for the model dependency is assumed in analogy to the case of calcium where a comparison of model dependent and model independent differences of radii is possible [2]. We assume an additional statistical error of $12 \%$ for the former and $6 \%$ for the latter value in accordance with the relative statistical errors of the model independent Barrett equivalent radii given in [2]. Having two independent values for $\delta\left\langle r^{2}\right\rangle^{38, A}$ and the measured isotope shifts $\delta \nu^{38,36}$ and $\delta \nu^{38,40}$, one obtains from eqs. (9) and (10) both the constant $C$ and the electronic factor:

$$
\begin{gathered}
C=-26.5[2.1] \mathrm{GHz} \text { a.m.u. } \\
F=101[37] \mathrm{MHz} / \mathrm{fm}^{2} .
\end{gathered}
$$

The errors arise almost exclusively from the estimated errors of the radii deduced from muonic X-ray data, and by the definition of the constant $C$ the voltage calibration error of the isotope shifts has been eliminated from the field shifts.

A further reduction of the errors is achieved by an independent evaluation of the electronic factor $F$ following the well established semi-empirical approaches outlined in [34]. From the magnetic hyperfine structure, neglecting screening effects and assuming the conventional $10 \%$ error, one obtains in good consistency with eq. (12)

$$
F=104[10] \mathrm{MHz} / \mathrm{fm}^{2} .
$$

With this semi-empirical electronic factor the average of the two possible solutions of eqs. (9) and (10) - using the isotope pairs 38, 36 and 38, 40, respectively - yields an improved value for the constant $C$ :

$$
C=M_{s}+k_{U}+k_{\Delta U}=26.3[1.2] \mathrm{GHz} \text { a.m.u. . }
$$

Here again the error is exclusively determined by the uncertainties of the muonic radii and the semi-empirical electronic factor. The dominating experimental systematic error 
arising from the voltage measurement only affects the (specific) mass effect. Of course, the specific mass shift constant according to eq. (7) is numerically identical with the constant $C$,

$$
M_{s}^{e x p}=-26.3[2.6] \mathrm{GHz} \text { a.m.u., }
$$

but it contains the additional error of the isotope shifts which is due to the voltage calibration. With the knowledge of $C$ the separation of the field shift from the experimentally determined isotope shift by means of eqs. (9) and (10) is possible for each isotope, independent of the actual composition of this constant. The values of $\delta\left\langle r^{2}\right\rangle^{38, A}$ compiled in Table 3 have been derived using the scaling factors (13) and (14)).

Table 3: Isotope shifts and changes in mean square nuclear charge radii. Errors in parentheses are purely statistical. The errors quoted in square brackets are systematic errors.

\begin{tabular}{|c|c|c|}
\hline$A$ & $\delta \nu^{38, A}[\mathrm{MHz}]$ & $\delta\left\langle r^{2}\right\rangle^{38, A}\left[\mathrm{fm}^{2}\right]$ \\
\hline 32 & $-881.5(4.0)[13.0]$ & $-0.375(38)[96]$ \\
33 & $-703.2(2.1)[10.5]$ & $-0.395(21)[87]$ \\
34 & $-552.6(0.7)[8.2]$ & $-0.251(6)[62]$ \\
35 & $-394.4(2.7)[5.9]$ & $-0.263(26)[53]$ \\
36 & $-265.4(0.4)[3.9]$ & $-0.082(4)[26]$ \\
37 & $-124.2(1.1)[1.9]$ & $-0.081(9)[17]$ \\
38 & 0 & 0 \\
39 & $122.4(7.0)[1.8]$ & $0.044(67)[12]$ \\
40 & $229.5(0.4)[3.5]$ & $0.169(4)[33]$ \\
46 & $835.2(2.3)[12.1]$ & $0.237(22)[78]$ \\
\hline
\end{tabular}

\section{Discussion}

\subsection{Nuclear moments far from stability: A test of the shell model}

\subsection{1 sd-shell}

The electromagnetic moments of the radioactive argon nuclei ${ }^{33} \mathrm{Ar},{ }^{35} \mathrm{Ar}$ and ${ }^{37} \mathrm{Ar}$ can be discussed within the framework of shell-model calculations for the sd-shell by Brown and Wildenthal [14]. This model assumes an inert ${ }^{16} \mathrm{O}$ core and the valence nucleons distributed over the full sd-shell. The model-independent effective interaction (Winteraction) is constructed from a complete set of single-particle energies and two-body interaction matrix elements adjusted to experimental binding energy data in a least-square fitting procedure [15]. As usual a renormalization of the free-nucleon operators is required, accounting for the effects of excluded configurations and sub-nucleonic degrees of freedom. The effective M1 and GT operators for a single-particle wave function for instance are expressed in terms of corrections involving the three general rank-one operators $\mathbf{s}, \mathbf{l}$ and $\mathbf{p}=(8 \pi)^{1 / 2}\left[\mathbf{Y}^{(2)} \otimes \mathbf{s}\right]^{(1)}$ and the use of empirically determined effective $g$-factors [14]. Both the empirically determined W-interaction and the effective $g$-factors are fairly well reproduced by calculations starting from more fundamental considerations $[14,38]$. The 
predictions for the magnetic moments and quadrupole moments are compiled in Table 4 . A graphical comparison between experiment and theory is given in Figures 5 and 6 .

Magnetic moments: A remarkably good agreement between theory and experiment is found for the magnetic moments of all neutron deficient argon nuclei including the new value of ${ }^{33} \mathrm{Ar}$ (see Figure 5). In particular the moment of ${ }^{33} \mathrm{Ar}$ appears to be sensitive to the choice of the effective $g$-factors. The latest adjustment [39] was preferred to the one in [40] (yielding $\mu=-0.831 \mu_{N}$ for ${ }^{33} \mathrm{Ar}$ ) which was based on rather poor empirical data defining the quenching for the $2 \mathrm{~s}_{1 / 2}$ orbital. Though the Schmidt (single-particle) limit $\left(\mu_{s p}=1.148 \mu_{N}\right.$ for a $\mathrm{d}_{3 / 2}$ neutron and $\mu_{s p}=-1.91 \mu_{N}$ for a $\mathrm{s}_{1 / 2}$ neutron) is almost reached for ${ }^{37} \mathrm{Ar}$, the results for ${ }^{33} \mathrm{Ar}$ and ${ }^{35} \mathrm{Ar}$ deviate strongly from the Schmidt limit. The case of short-lived ${ }^{33} \operatorname{Ar}\left(T_{z}=-3 / 2\right)$ fits perfectly to the overall trend of good consistency observed for the upper sd-shell nuclei closer to stability (see Figure 5).

Quadrupole moments: Since the experimental values of quadrupole moments have larger errors and often the absolute calibration (depending on atomic properties) is subject to uncertainties of about $10 \%$, the comparison between theory and experiment is less quantitative than for the magnetic moments. The quadrupole moments were calculated using the shell model with the standard effective charges of $1.35 e$ for the proton and $0.35 e$ for the neutron [14] and with harmonic-oscillator radial wave functions ( $\hbar \omega=$ $\left.45 A^{-3 / 2}-25 A^{-1 / 3}\right)$. The predictive power of the shell model for quadrupole moments is excellent - for the radioactive argon nuclei (see Table 4) as well as for other nuclear ground states all over the sd-shell (see Figure 6). Even the cases of considerable ground state correlation, indicated by quadrupole moments of several single particle units, are quite well reproduced. Contrary to the magnetic dipole operator, the electric quadrupole operator is rather sensitive to higher-order configuration mixing ( $2 \hbar \omega$-admixtures).

Table 4: Predictions for orbit-, spin- and tensor-components of the magnetic moment [39], the resulting magnetic moments, and the quadrupole moments of argon nuclei in the sd-shell model and of ${ }^{39} \mathrm{Ar}$ with a mixed sd-fp configuration [42].

\begin{tabular}{|c|c|c|c|c|c|c|c|c|}
\hline & $I$ & $\alpha_{l}\left[\mu_{N}\right]$ & $\alpha_{s}\left[\mu_{N}\right]$ & $\alpha_{p}\left[\mu_{N}\right]$ & $\mu^{t h}\left[\mu_{N}\right]$ & $\mu^{\text {exp }}\left[\mu_{N}\right]$ & $Q^{\text {th }}[\mathrm{mb}]$ & $Q^{\text {exp }}[\mathrm{mb}]$ \\
\hline${ }^{33} \mathrm{Ar}$ & $1 / 2$ & 0.038 & -0.716 & -0.062 & -0.739 & $-0.723(6)$ & - & - \\
${ }^{35} \mathrm{Ar}$ & $3 / 2$ & 0.161 & 0.368 & 0.160 & 0.688 & $0.633(2)$ & -88.7 & $-84(7)[8]$ \\
${ }^{37} \mathrm{Ar}$ & $3 / 2$ & 0.270 & 0.713 & 0.172 & 1.155 & $1.145(5)$ & 76.1 & $76.2(1.6)[7.6]$ \\
${ }^{39} \mathrm{Ar}$ & $7 / 2$ & -1.847 & 0.104 & - & -1.436 & $-1.588(15)$ & -128 & $-117(20)[12]$ \\
\hline
\end{tabular}

\subsection{2 fp-shell}

For ${ }^{39} \mathrm{Ar}$ the calculation is based on an effective interaction developed for the nuclei with mixed sd-fp configurations [42]. Employing the free-nucleon $g$-factors the magnetic moment of ${ }^{39} \mathrm{Ar}$ is obtained to be $\mu=-1.743 \mu_{N}\left(\alpha_{s}=0.104 \mu_{N}, \alpha_{l}=-1.847 \mu_{N}\right)$. The result obtained using the effective sd M1 operator from [39] and the effective fp M1 operator from [43] is $-1.436 \mu_{N}$. The experimental value lies in between the free and effective operator results. It is interesting to note that the experimental ${ }^{39} \mathrm{Ar}$ and ${ }^{41} \mathrm{Ca}$ magnetic moments are nearly equal, $-1.588(15) \mu_{N}$ and $-1.595 \mu_{N}$, respectively. The calculated quadrupole moment of $-128 \mathrm{mb}$ (obtained with the standard effective charges 
of $1.35 \mathrm{e}$ for the proton and $0.35 \mathrm{e}$ for the neutron) is in good agreement with experiment, too.

\subsection{Nuclear shape and radii}

Within the statistical errors the evolution of the mean square charge radii of argon isotopes as a function of the neutron number (see Figure 7) is characterized by:

(i) an almost linear increase of the radii of even isotopes between $N=14$ and $N=22$;

(ii) a "normal" odd-even staggering effect for the sd-shell nuclei;

(iii) a slope change in the increase of the charge radii within the $f_{7 / 2}$-shell indicated by the fact that the radii of ${ }^{40} \operatorname{Ar}(N=22)$ and ${ }^{46} \operatorname{Ar}(N=28)$ are almost the same.

These features are insensitive to the calibration uncertainty affecting only the overall scale of the changes in the radii. The most striking characteristic of the argon data is the absence of any shell effect at the $N=20$ shell closure. This is in contrast to the observation of pronounced changes in the $N$-dependence of the mean square radii at all spherical shell closures with $N \geq 28[44,13,34]$ - from a small decrease or increase below to a steep increase above.

Part of this effect is usually attributed to changes in the shape of the nuclei. In the uniform-density sharp-surface approximation the mean square charge radius is given up to the lowest order in deformation $\beta_{i}$ by

$$
\left\langle r^{2}\right\rangle=\left\langle r^{2}\right\rangle_{s p h}\left(1+\frac{5}{4 \pi} \sum_{i}\left\langle\beta_{i}^{2}\right\rangle\right) .
$$

Thus the quantity $\left\langle r^{2}\right\rangle$ probes contributions of both static and dynamic multipole deformations of the nuclear surface of multipolarity $i \geq 2$. The mean square quadrupole deformation $\left(\left\langle\beta^{2}\right\rangle \equiv\left\langle\beta_{2}^{2}\right\rangle\right)$ is considered to be the dominant contribution $[9,44,34]$. The finite range droplet model $[45,46]$ provides a globally good description of the dominant spherical (volume) contribution $\left(\left\langle r^{2}\right\rangle_{s p h}\right)$ to the mean square charge radii. Assuming nearly constant shape for the sequence of argon isotopes, the droplet model line in Figure 7 reproduces well the increase of the radii up between $N=14$ and 22, but it obviously fails for the $\mathrm{f}_{7 / 2}$-shell between $N=22$ and $N=28$.

In the microscopic view discontinuities in the volume contribution $\left\langle r^{2}\right\rangle_{s p h}$ arise from a monopole polarization of the proton core by the additional valence neutrons. Just above a closed neutron (sub-)shell the radii of the valence neutron orbits are larger than the average radii of the filled proton and neutron orbits. Due to the attractive protonneutron interaction, the energy is minimized when neutrons and protons overlap as much as possible, which is achieved by a 'pulling out' of the core protons. Another way in which the addition of valence neutrons changes the proton density is when the neutrons are added in an orbital which has an interior node (such as the $2 \mathrm{~s}$ and $2 \mathrm{p}$ orbitals). In this case the saturating effect for the mean field forces the entire density (all protons and neutrons) to expand in order to keep the interior density approximately constant. Both of these types of monopole polarization come out of a Skyrme-type Hartree-Fock calculation because of the self-consistency between the density and the potential [9]. 
In the present case the spherical Hartree-Fock calculations for the mean square radii were carried out with the SGII Skyrme interaction [47]. This interaction reasonably well reproduces the single-particle energies near ${ }^{40} \mathrm{Ca}$ and their mass dependence up to ${ }^{48} \mathrm{Ca}$. In particular, it is known that the $1 \mathrm{~d}_{3 / 2}$ and $2 \mathrm{~s}_{1 / 2}$ single particle spacing changes from $2.5 \mathrm{MeV}$ in ${ }^{39} \mathrm{~K}$ to $-0.36 \mathrm{MeV}$ in ${ }^{47} \mathrm{~K}$, and this cross-over is reproduced by the SGII interaction. Without a residual interaction, the two sd-shell proton holes in the argon isotopes change from two holes in the $1 \mathrm{~d}_{3 / 2}$ orbital in ${ }^{38} \mathrm{Ar}$ to two holes in the $2 \mathrm{~s}_{1 / 2}$ orbital in ${ }^{46} \mathrm{Ar}$. With a residual interaction, the two holes in ${ }^{38} \mathrm{Ar}$ are still mainly in the $1 \mathrm{~d}_{3 / 2}$ orbital because of the large $1 \mathrm{~d}_{3 / 2}-2 \mathrm{~s}_{1 / 2}$ single-particle energy gap. But in ${ }^{46} \mathrm{Ar}$ the two holes are split between $1 \mathrm{~d}_{3 / 2}$ and $2 \mathrm{~s}_{1 / 2}$ - a shell-model calculation for the two-hole spectrum of ${ }^{46} \mathrm{Ar}$ indicates that there is approximately one hole in $1 \mathrm{~d}_{3 / 2}$ and one hole in $2 \mathrm{~s}_{1 / 2}$. Thus we have carried out the Hartree-Fock calculations with the constraint on the two-proton-hole occupancies obtained from a linear interpolation between ${ }^{38} \mathrm{Ar}$ and ${ }^{46} \mathrm{Ar}$. The proton orbit occupancies affect the Hartree-Fock radii in a subtle way related to the interplay between the saturation properties of the Skyrme interaction and the difference in the radial structure of the orbits. It turns out that moving the proton hole from $1 \mathrm{~d}_{3 / 2}$ to $2 \mathrm{~s}_{1 / 2}$ has the effect of increasing the radius. The relatively larger change from ${ }^{38} \mathrm{Ar}$ to ${ }^{46} \mathrm{Ar}$ compared to the change from ${ }^{40} \mathrm{Ca}$ to ${ }^{48} \mathrm{Ca}$ (see Figure 9) appears to agree with the calculations. The results for the potassium isotopes are intermediate to those for the argon and calcium isotopes with the difference that the proton hole occupancy changes abruptly from one hole in the $1 \mathrm{~d}_{3 / 2}$ to the one hole in the $2 \mathrm{~s}_{1 / 2}$ at about $N=27$. In addition to these spherical effects, the deformation effects will enlarge the mean square radii away from the ${ }^{40} \mathrm{Ca}$ and ${ }^{48} \mathrm{Ca}$ closed shell as indicated by equation (16).

Beyond $N=28$ the spherical Hartree-Fock calculations show a jump which is related to the neutron occupancy of the $2 \mathrm{p}_{3 / 2}$ orbital. The reason for this is that between $N=20$ and $N=28$ the neutrons occupy the $1 \mathrm{f}_{7 / 2}$ orbital whose density is concentrated at the surface, whereas between $N=28$ and $N=32$ the neutrons start to occupy the $2 \mathrm{p}_{3 / 2}$ orbital which has an interior node. Due to the (density) saturation property, this interior node has the effect of pushing out all of the nucleons including the core protons which are responsible for an increase of the charge radii.

Other mean-field calculations for the mean square charge radii have recently been reported by Werner et al. [49]. Their results with the SIII interaction are similar to the SGII results discussed above. Their relativistic mean field results give a smaller increase as a function of neutron number which is inconsistent with the present data. (We also note that the experimental data for the argon isotopes used in ref. [49] should be replaced by the present results.)

Part of the remaining discrepancy between theory and experiment can be attributed to quadrupole core polarization which for the even- $A$ argon nuclei is considered by a semi-empirical shape correction of the form (16) to the HF prediction $\left(\left\langle r^{2}\right\rangle_{s p h}\right)$. The ratio between the reduced transition strength $B\left(\mathrm{E} 2 ; 0^{+} \rightarrow 2^{+}\right.$) (taken from [50]) and the mean square charge radius (taken from Table 3 in combination with [2]) may serve as a measure for the root mean square quadrupole deformation $\left(\left\langle\beta^{2}\right\rangle^{1 / 2}\right)$. The approximate relation [51]

$$
\left\langle\beta^{2}\right\rangle^{1 / 2}=\frac{4 \pi}{5} \frac{\left[B\left(\mathrm{E} 2 ; 0^{+} \rightarrow 2^{+}\right)\right]^{1 / 2}}{Z\left\langle r^{2}\right\rangle}
$$


suggests $\left\langle\beta^{2}\right\rangle^{1 / 2} \approx 0.2$ for $N=16,18$ and 22 , while a minimum of $\left\langle\beta^{2}\right\rangle^{1 / 2} \approx 0.14$ is reached at $N=20$. The corresponding shape correction to spherical (HF) radii implies a rather small shell effect at the $N=20$ shell closure and results in an almost quantitative agreement between theory and experiment (see Figure 8).

For the odd- $A$ nuclei a relation between the spectroscopic quadrupole moment $\left(Q_{s}\right)$ and the static intrinsic quadrupole moment $\left\langle Q_{0}\right\rangle$, measuring the static nuclear deformation $\left\langle\beta_{2}\right\rangle$ by [51]

$$
\left\langle\beta_{2}\right\rangle=\frac{\sqrt{\pi / 5}}{Z} \frac{\left\langle Q_{0}\right\rangle}{\left\langle r^{2}\right\rangle},
$$

involves the introduction of a model specifying the coupling between nuclear single-particle and collective motion [52]. A simple relation only holds in the strong coupling limit for a permanently deformed, axially symmetric nucleus. However, the relatively small quadrupole moments $\left(Q_{s}\right.$ in Table 2$)$ indicate near-spherical equilibrium shapes for the ground states. This is confirmed by the success of the spherical shell-model calculation presented in Section 4.1. Moreover, the experimental information about the strength of collective quadrupole (E2) vibrations required to specify the non-permanent quadrupole deformation is not available, which means that no semi-empirical shape correction to the radii can be derived for the odd- $A$ argon isotopes.

\section{Conclusion}

The hyperfine structure and isotope shift measurements on ${ }^{32-40} \mathrm{Ar}$ and ${ }^{46} \mathrm{Ar}$ are the first optical high-resolution investigations of argon, and they cover a whole series of radioactive isotopes. The study of isotopes far from stability has become possible due to the ultrasensitive resonance detection technique combining optical pumping and state-selective collisional ionization with an isotope-selective detection of the ions based on the $\beta$-decay.

The results comprise magnetic dipole and electric quadrupole moments of the odd- $A$ isotopes which were employed as a test of shell-model calculations for the sd- and fp-shell. A very good agreement between the experimental results and the shell-model predictions (including those for ${ }^{33} \mathrm{Ar}$ ) is found.

Since the dominating isovector part of the M1 operator is related to the GT operator via isospin rotational invariance, there is a strong similarity between the gross features of magnetic moments and the Gamow-Teller decay properties. In case of ${ }^{33} \mathrm{Ar}$ the experimental Gamow-Teller sum strength was found to be at variance with the shellmodel calculation [16]. However, our direct measurement of the magnetic moment of ${ }^{33} \mathrm{Ar}$ - the first one for an sd-shell nucleus with $T_{z}=-3 / 2-$ is in excellent agreement with the shell-model prediction. As mentioned in the introduction, the problem with the Gamow-Teller strength distribution appears to be concentrated in the centroid energy of the Gamow-Teller giant resonances [18]. In spite of this, the W-interaction does quite well in reproducing the magnetic moments.

The magnetic moment is known to be particularly sensitive to details of the nuclear interaction. The additional information provided by magnetic moments was recently employed for an empirical determination of the effective interaction for the 1p-shell [53]. Thus we conclude that the systematics of the electromagnetic moments proves the good predictive power of the sd-shell calculations of Brown and Wildenthal with the W-interaction for short-lived nuclei in the upper sd-shell. 
Further results comprise the changes in mean square charge radii of argon isotopes between $N=14$ and $N=28$. For the even- $A$ isotopes between $N=14$ and $N=22$ an almost linear increase of the radii is observed. The odd-even staggering effect in this region has the usual sign and magnitude.

The disappearance of the shell effect in the radii across the $N=20$ shell closure is explained by a cancellation of the monopole polarization and the quadrupole polarization of the proton core under successive addition of valence neutrons. The effect of monopole core polarization was calculated using a (SGII) Skyrme-type HF model. Quadrupole core polarization was considered by a semi-empirical correction based on the properties of the lowest $2^{+}$-states of the doubly-even isotopes.

The present data on argon may serve as a cornerstone for a more global systematics of the nuclear radii in the region of calcium (see Figure 9). A suitable production target should make it possible to extend the measurements of radii to the neutron-rich argon nuclei in the $1 \mathrm{f}_{7 / 2^{-}}$and $2 \mathrm{p}_{3 / 2^{-}}$-shell. This should allow the study of several shell closures and a comparison to the existing data on potassium and calcium. There is already a remarkable and so far unexplained difference in the parabolic gross behaviour and the odd-even staggering between the radii of the $\mathrm{Ca}$ and the $\mathrm{K}$ isotopes in the $\mathrm{f}_{7 / 2}$-shell $(20 \leq N \leq 28)$.

Further investigations will have to be devoted to the role of higher-order core polarization [10] and/or particle excitation from the sd- to the fp-shell [8, 9], which has been suggested to contribute considerably to the parabolic $N$-dependence of the radii of calcium isotopes.

\section{Acknowledgments}

This work has been funded by the German Ministry for Education, Science, Research and Technology (BMBF) under the contracts $06 \mathrm{MZ} 501 \mathrm{I}$ and $06 \mathrm{MZ}$ 566. PL and LV are Senior Research Assistants of the Belgian National Fund for Scientific Research (N.F.W.O.). BAB would like to acknowledge support from NSF grant PHY94-03666 and from the Alexander von Humboldt Foundation. 


\section{References}

[1] H.D. Wohlfahrt, E.B. Shera, M.V. Hoehn, Y. Yamazaki and R.M. Steffen, Phys. Rev. C 23 (1981) 533

[2] G. Fricke, C. Bernhardt, K. Heilig, L.A. Schaller, L. Schellenberg, E.B. Shera and C.W. de Jager, At. Data Nucl. Data Tables 60 (1995) 177

[3] H.J. Emrich, G. Fricke, G. Mallot, H. Miska, H.-G. Sieberling, J.M. Cavedon, B. Frois and D. Goutte, Nucl. Phys. A 396 (1983) 401c

[4] C.W.P. Palmer, P.E.G. Baird, S.A. Blundell, J.R. Brandenberger, C.J. Foot, D.N. Stacey and G.K. Woodgate, J. Phys. B 17 (1984) 2197

[5] A.-M. Mårtensson-Pendrill, A. Ynnermann, H. Warston, L. Vermeeren, R.E. Silverans, A. Klein, R. Neugart, Ch. Schulz, P. Lievens and ISOLDE Collaboration, Phys. Rev. A 45 (1992) 4675

[6] E. Caurier and A. Poves, Nucl. Phys. A 385 (1982) 407

[7] M. Waroquier, K. Heyde and G. Wenes, Nucl. Phys. A 404 (1983) 269.

[8] B.A. Brown, S.E. Massen and P.E. Hodgson, Phys. Lett. 85B (1979) 167

[9] B.A. Brown, S.E. Massen and P.E. Hodgson, J. Phys. G5 (1979) 1655

[10] F. Barranco and R.A. Broglia, Phys. Lett. 151B (1985) 90

[11] F. Touchard, P. Guimbal, S. Büttgenbach, R. Klapisch, M. de Saint Simon, J.M. Serre, C. Thibault, H.T. Duong, P. Juncar, S. Liberman, J. Pinard and J.L. Vialle, Phys. Lett. 108B (1982) 169

[12] L. Vermeeren, P. Lievens, R.E. Silverans, U. Georg, M. Keim, A. Klein, R. Neugart, M. Neuroth, F. Buchinger and ISOLDE Collaboration: The mean square nuclear charge radius of ${ }^{39} \mathrm{Ca}$, submitted to J. Phys. G

[13] L. Vermeeren, R.E. Silverans, P. Lievens, A. Klein, R. Neugart, Ch. Schulz and F. Buchinger, Phys. Rev. Lett. 68 (1992) 1679

[14] B.A. Brown and B.H. Wildenthal, Ann. Rev. Nucl. Part. Sci. 38 (1988) 29

[15] B.H. Wildenthal, Prog. Part. Nucl. Phys. 11 (1984) 5

[16] M.J.G. Borge, P.G. Hansen, B. Jonson, S. Mattsson, G. Nyman, A. Richter, K. Riisager and ISOLDE Collaboration, Z. Phys. A 332 (1989) 413

[17] E.G. Adelberger, A. Garcia, P.V. Magnus and D.P. Wells, Phys. Rev. Lett. 67 (1991) 3658; A. Garcia et al., Phys. Rev. Lett. 67 (1991) 3654; W. Trinder et al., Phys. Lett B349 (1995) 267

[18] B.A. Brown, Phys. Rev. Lett. 69 (1992) 1034; Proceedings of the IV International Symposium on Weak and Electromagnetic Interactions in Nuclei, 12-16 June, 1995, Osaka, Japan, edited by H. Ejiri, T. Kishimoto and T. Sato, (World Scientific, 1996) 281. 
[19] B.A. Brown and B.H. Wildenthal, At. Data Nucl. Data Tables 33 (1985) 347

[20] R. Neugart, in Progress in Atomic Spectroscopy, Part D, eds. H. J. Beyer and H. Kleinpoppen, (Plenum Press, New York, 1987) p.75

[21] E. Kugler, D. Fiander, B. Jonson, H. Haas, A. Przewloka, H.L. Ravn, D.J. Simon, K. Zimmer and ISOLDE Collaboration, Nucl. Instr. Meth. B 70 (1992) 41

[22] R. Neugart, W. Klempt and K. Wendt, Nucl. Instr. Meth. B 17 (1986) 354

[23] T. Björnstad, M.J.G. Borge, P. Dessagne, R.-D. von Dincklage, G.T. Ewan, P.G. Hansen, A. Huck, B. Jonson, G. Klotz, A. Knipper, P.O. Larsson, G. Nyman, H.L. Ravn, C. Richard-Serre, K. Riisager, D. Schardt, G. Walter and ISOLDE Collaboration, Nucl. Phys. A 443 (1985) 283

[24] A. Klein, Thesis, Mainz (1995) and to be published

[25] G.P. Barwood, P. Gill and W.R.C. Rowley, Appl. Phys. B 53 (1991) 142

[26] E. Arimondo, M. Inguscio and P. Violino, Rev. Mod. Phys. 49 (1977) 31

[27] M.M. Robertson, J.E. Mack and V.W. Cohen, Phys. Rev. 140 (1965) B 820

[28] W. Traub, F.L. Roesler, M.M. Robertson and V.W. Cohen, J. Opt. Soc. Am. 57 (1967) 1452

[29] M.L. Pitt, M. Jozoff, F.P. Calaprice, G. Wang and E.K. Warburton, Bull. Am. Phys. Soc. 33 (1988) 1564

[30] F.P. Calaprice, E.D. Commins and D.A. Dobson, Phys. Rev. 137 (1965) B 1453

[31] S. Büttgenbach, Hyperfine Interactions 20 (1984) 1

[32] A. Bohr and V.F. Weisskopf, Phys. Rev. 77 (1950) 94

[33] F.D. Feiock and W.R. Johnson, Phys. Rev. 187 (1969) 39

[34] M. Keim, E. Arnold, W. Borchers, U. Georg, A. Klein, R. Neugart, L. Vermeeren, R.E. Silverans and P. Lievens, Nucl. Phys. A 586 (1995) 219

[35] W. Borchers, E. Arnold, W. Neu, R. Neugart, K. Wendt, G. Ulm and ISOLDE Collaboration, Phys. Lett. B 216 (1989) 7

[36] S.A. Blundell, P.E.G. Baird, C.W.P. Palmer, D.N. Stacey, G.K. Woodgate and D. Zimmermann, Z. Phys. A 321 (1985) 31

[37] Z. Fang, O. Redi and H.H. Stroke, J. Phys. II France 2 (1992) 877

[38] J. Fiase, A. Hamoudi, J.M. Irvine and F. Yazici, J. Phys. G 14 (1988) 27

[39] B.A. Brown and B.H. Wildenthal, Nucl. Phys. A474 (1987) 290

[40] B.A. Brown and B.H. Wildenthal, Phys. Rev. C 28 (1983) 2397 
[41] M. Carchidi, B.H. Wildenthal and B.A. Brown, Phys. Rev. C 34 (1986) 2280

[42] E.K. Warburton, J.A. Becker and B.A. Brown, Phys. Rev. C 41 (1990) 1147

[43] W.A. Richter, M.G. Van der Merwe, R.E. Julies and B.A. Brown, Nucl. Phys. A 577 (1994) 585

[44] E.W. Otten, in Treatise on Heavy-Ion Science, Vol. 8, ed. D.A. Bromley, (Plenum Press, New York, 1989), p.517

[45] W.D. Myers and K.-H. Schmidt, Nucl. Phys. A 410 (1983) 61

[46] P. Möller, W.D. Myers, W.J. Swiatecki and J. Treiner, At. Data and Nucl. Data Tables 39 (1988) 225

[47] Nguyen Van Giai and H. Sagawa, Nucl. Phys. A 371 (1981) 1 ; Phys. Lett 106B (1981) 379

[48] A.-M. Mårtensson-Pendrill, L. Pendrill, S. Salomonson, A. Ynnermann and H. Warston, J. Phys. B 23 (1990) 1749

[49] T.R. Werner, J.A. Sheikh, M. Misu, W. Nazarewicz, J. Rikovska, K. Heeger, A.S. Umar and M.R. Strayer, Nucl. Phys. A 597 (1996) 327

[50] S. Raman, C.W. Nestor, jr., S. Kahane and K.H. Bhatt, At. Data Nucl. Data Tables $42(1989) 1$

[51] K. Kumar, Phys. Rev. Lett. 28 (1972) 249

[52] A. Bohr and B.R. Mottelson, Dan. Mat. Fys. Medd. 27 (1953) 1

[53] A.M.G. van Hees, A.A. Wolters and P.W.M. Glaudemans, Nucl. Phys. A 476 (1988) 61 


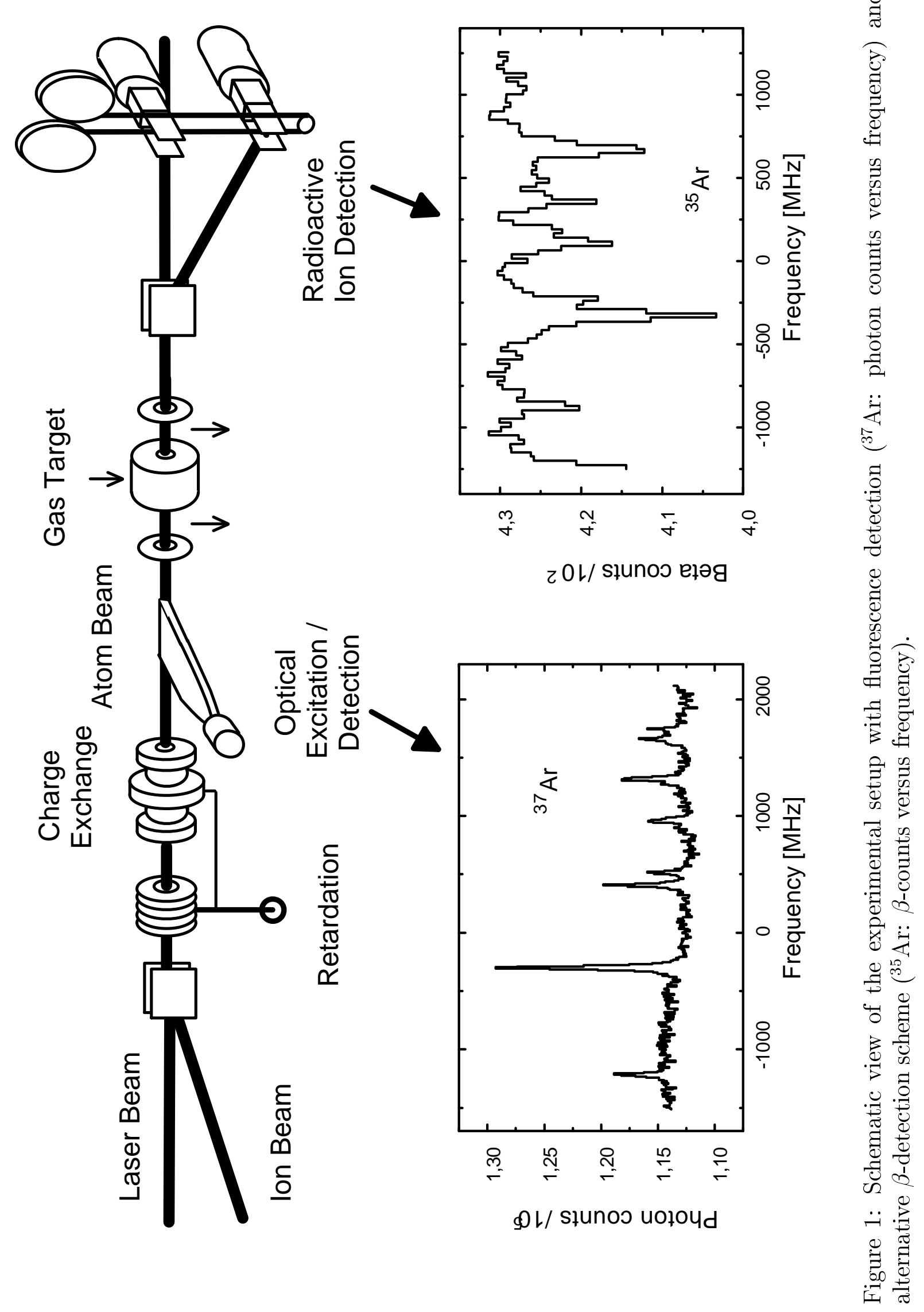




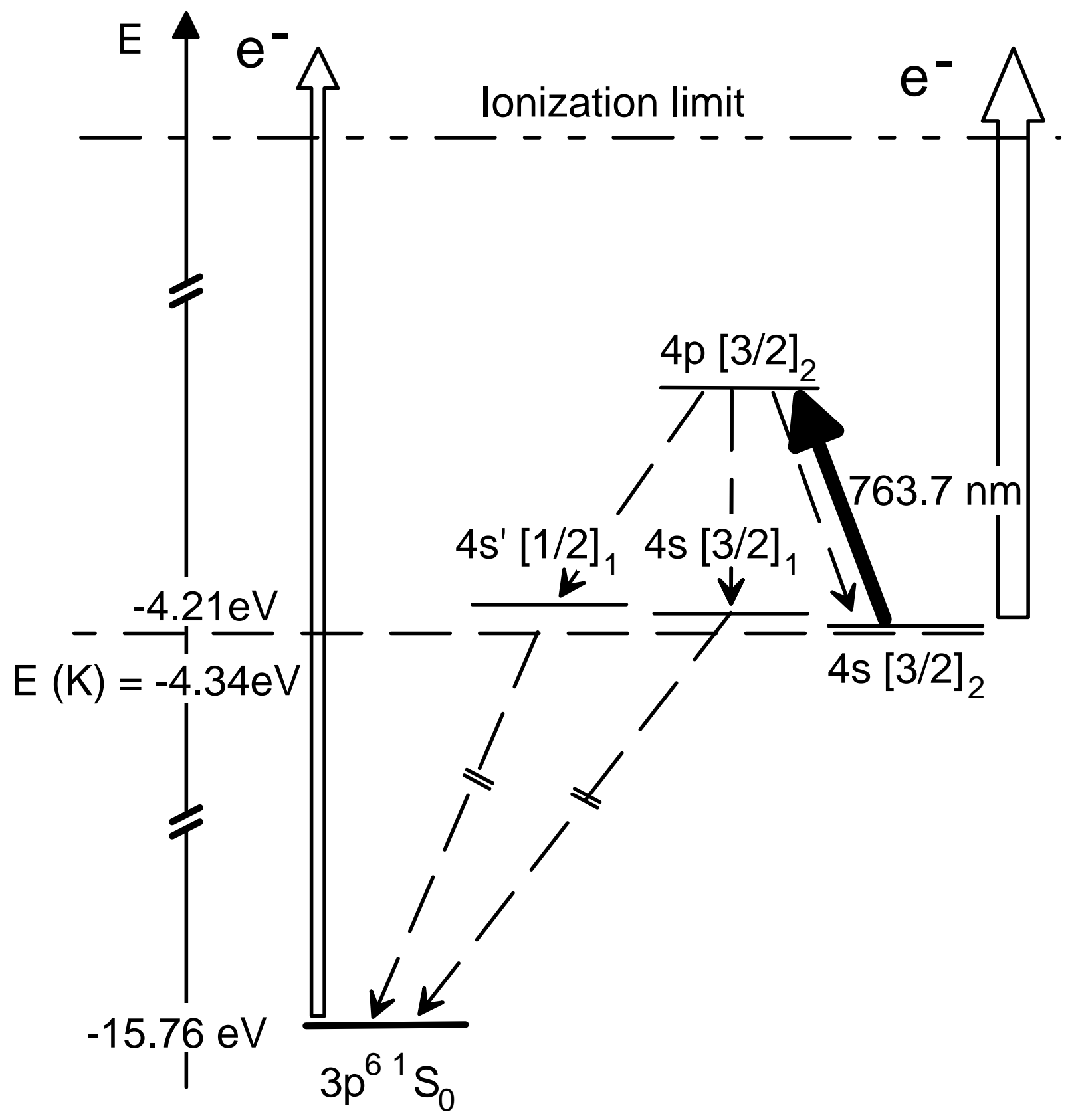

Figure 2: Partial atomic level scheme of argon, optical pumping cycle and ionization energy of the charge exchange medium potassium. 

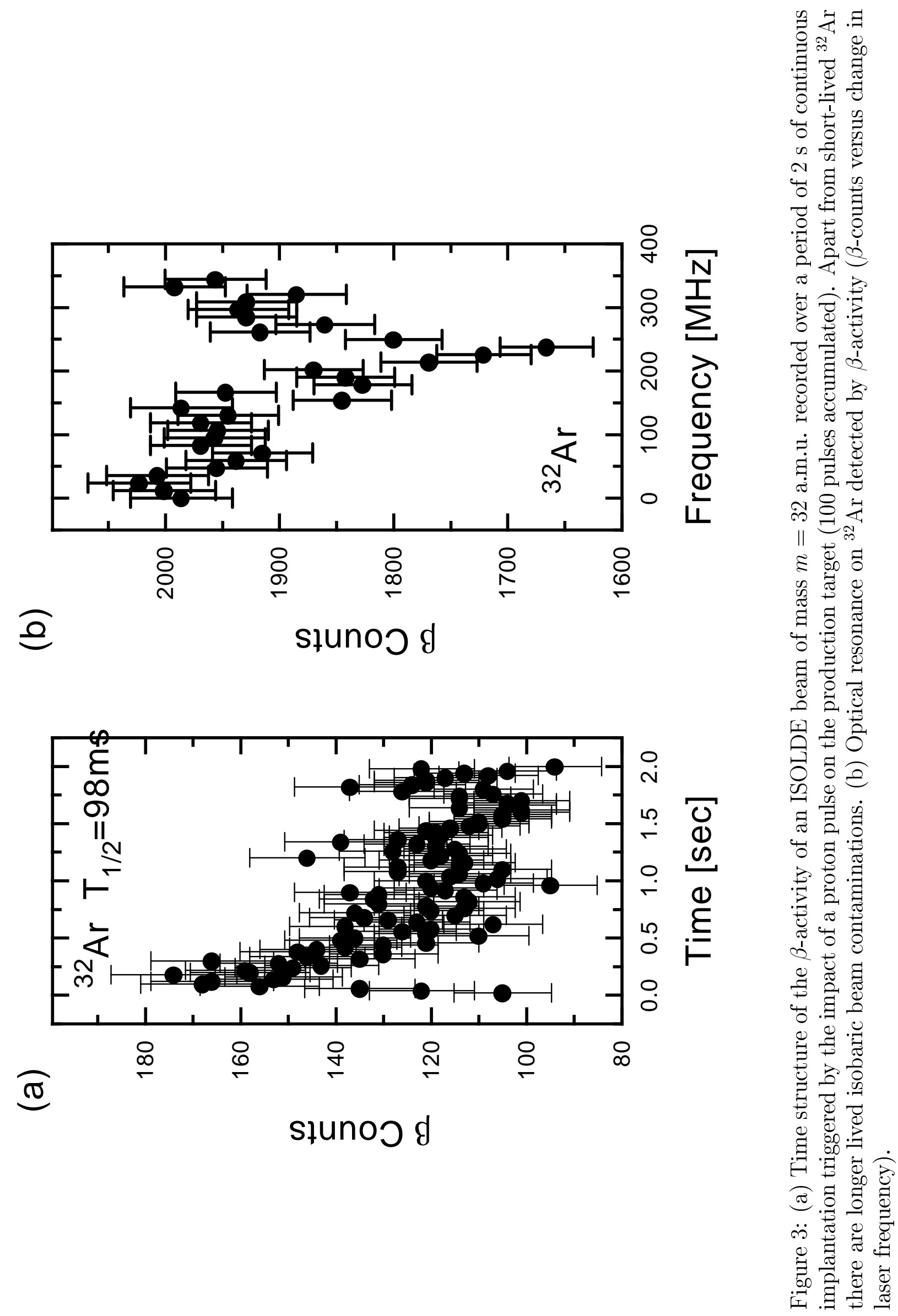


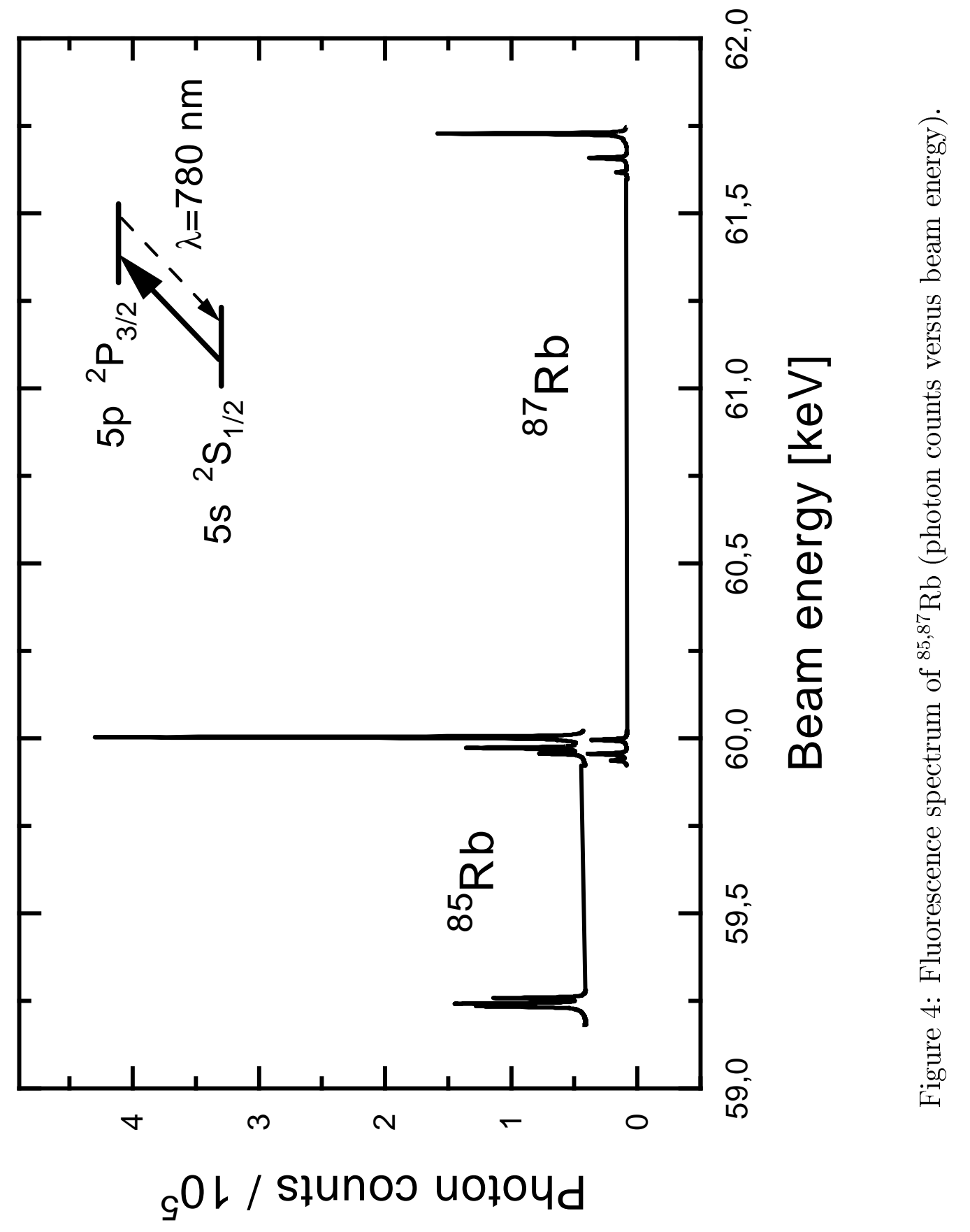




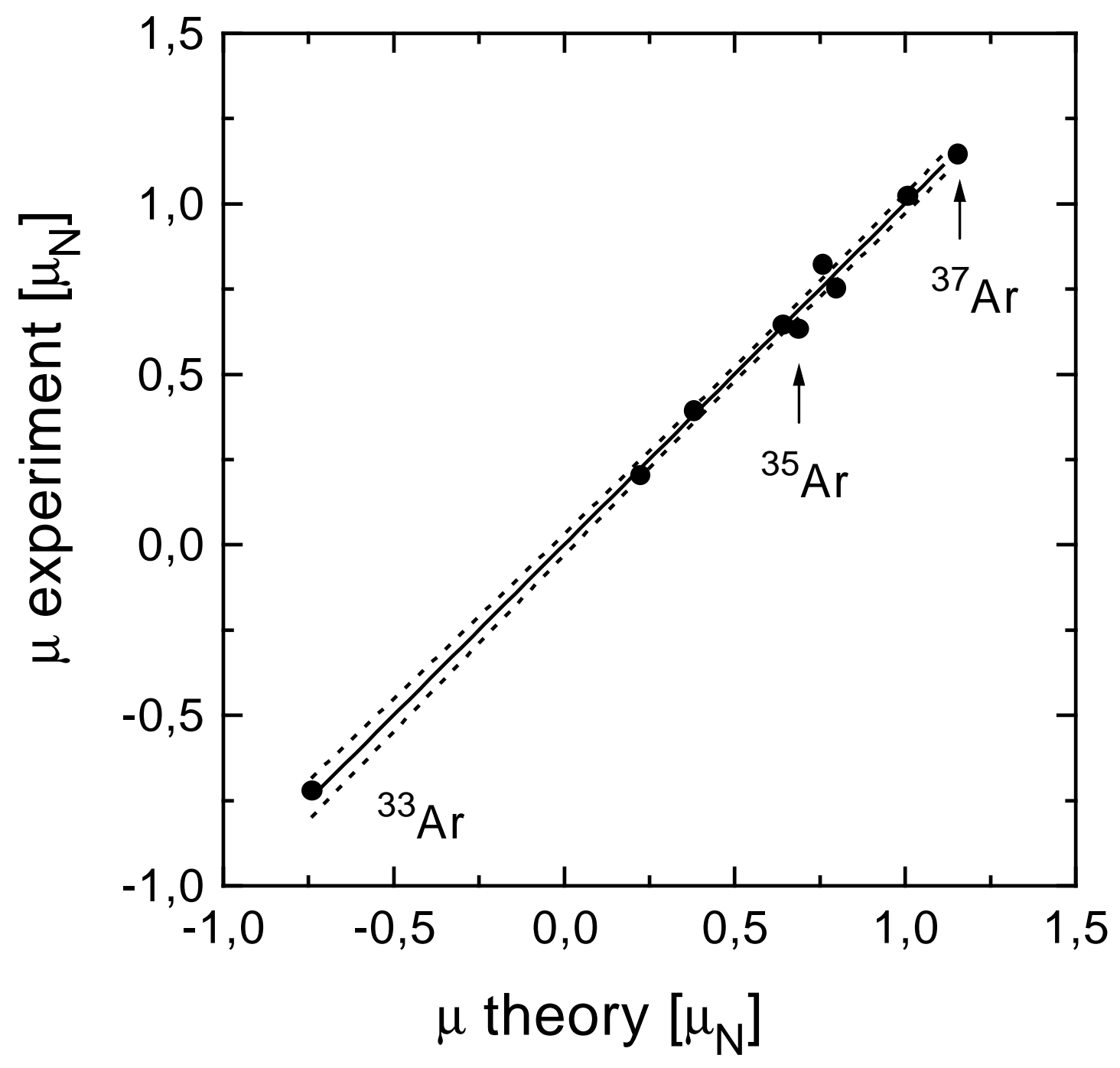

Figure 5: Experiment versus theory for magnetic moments in the upper sd-shell. Shown are the odd- $Z$ nuclei ${ }^{33} \mathrm{Cl},{ }^{35} \mathrm{Cl},{ }^{37} \mathrm{~K},{ }^{39} \mathrm{~K}$, the odd- $N$ nuclei ${ }^{33} \mathrm{~S},{ }^{39} \mathrm{Ca}$ and the marked argon isotopes. The solid line representing a linear regression to all nuclei except ${ }^{33} \mathrm{Ar}$ indicates perfect agreement between theory and experiment (slope $m=0.99(2)$ ). The dashed lines correspond to the $1 \sigma$ confidence band of the regression. 


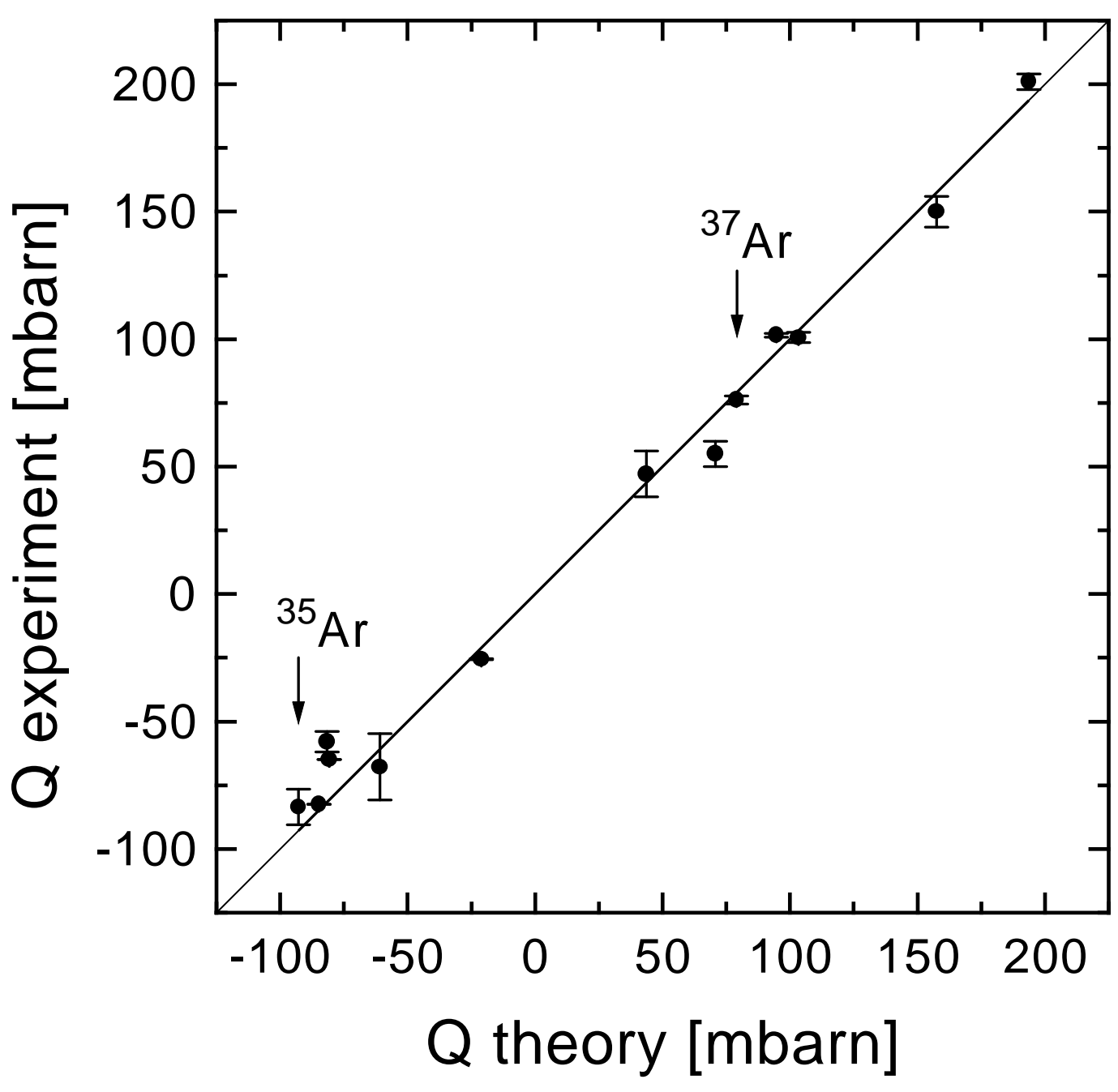

Figure 6: Experiment versus theory for quadrupole moments in the sd-shell. Shown are the ground states of the odd- $Z$ nuclei ${ }^{17} \mathrm{~F},{ }^{23} \mathrm{Na},{ }^{27} \mathrm{Al},{ }^{35} \mathrm{Cl},{ }^{37} \mathrm{Cl}$ and ${ }^{39} \mathrm{~K}$, the odd- $N$ nuclei ${ }^{17} \mathrm{O},{ }^{21} \mathrm{Ne},{ }^{25} \mathrm{Mg},{ }^{33} \mathrm{~S},{ }^{35} \mathrm{~S}$, and the marked argon isotopes. Earlier calculated values are from ref. [41]. For Ar isotopes only the statistical errors of the experimental values are indicated. 


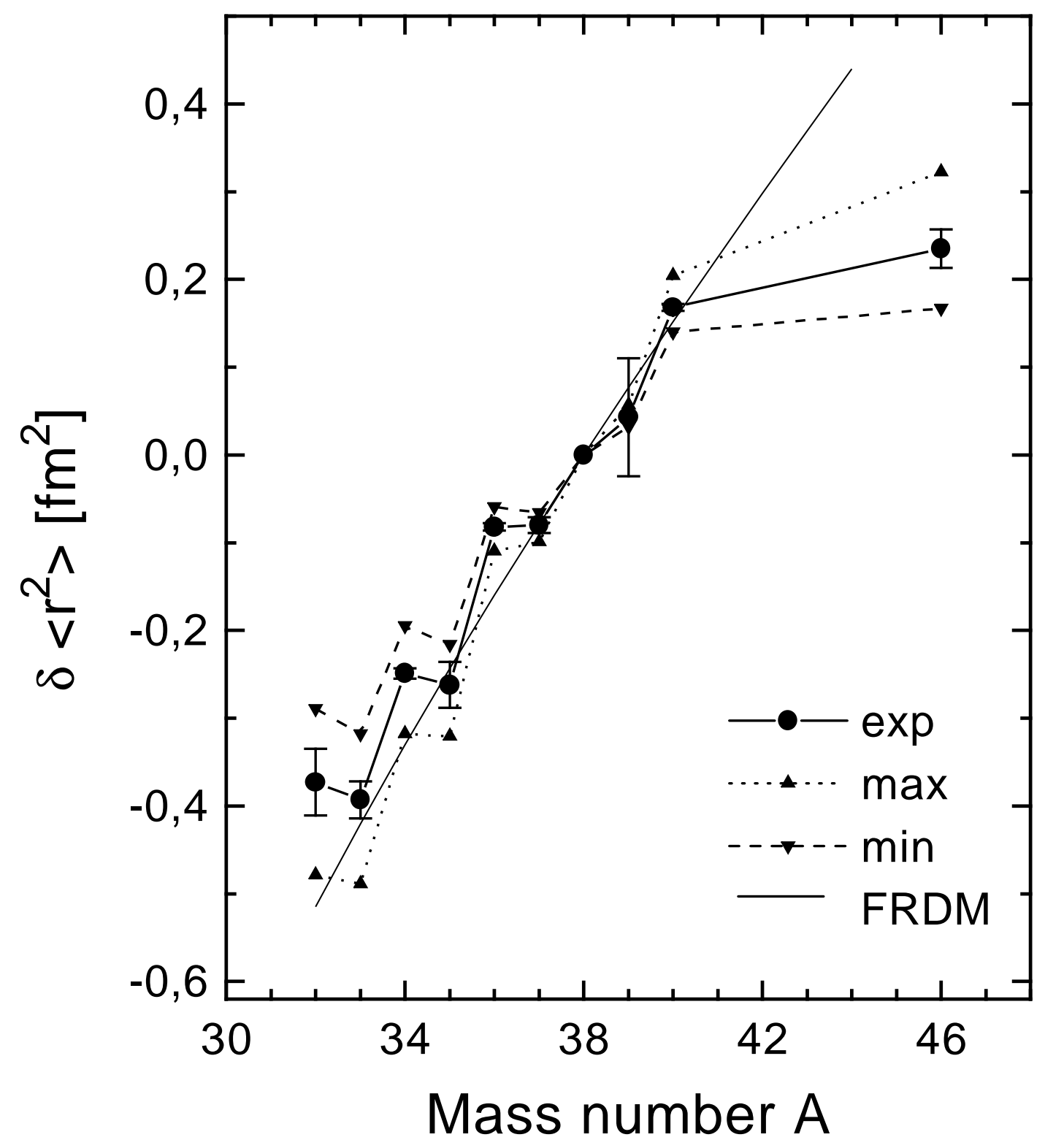

Figure 7: Changes in mean square charge radii $\left(\delta\left\langle r^{2}\right\rangle^{38, A}\right)$ versus neutron number (dots)). The error bars correspond to the statistical errors only. The results obtained by changing the calibration within the error limits are indicated by the up and down triangles. The prediction of the finite range droplet model $\left(\left\langle\beta^{2}\right\rangle^{1 / 2}=0\right)$ is given by the solid line. 


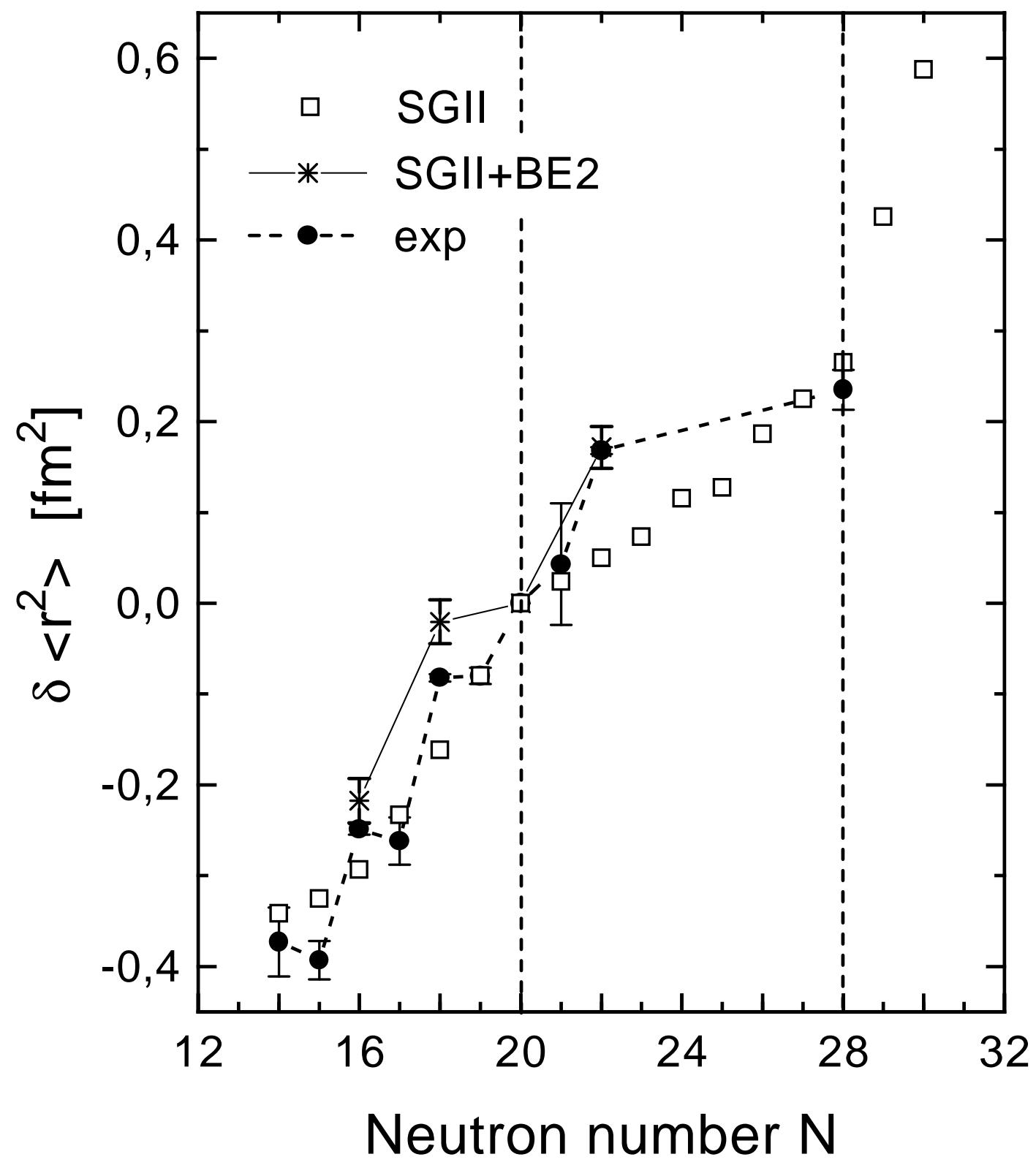

Figure 8: Comparison between experimental $\delta\left\langle r^{2}\right\rangle^{38, A}$ - values (dots) and a spherical Skyrme (SGII) Hartree-Fock calculation (squares). For the even- $A$ isotopes a semiempirical correction for quadrupole core polarization has been based on $B(\mathrm{E} 2)$ data (stars). 


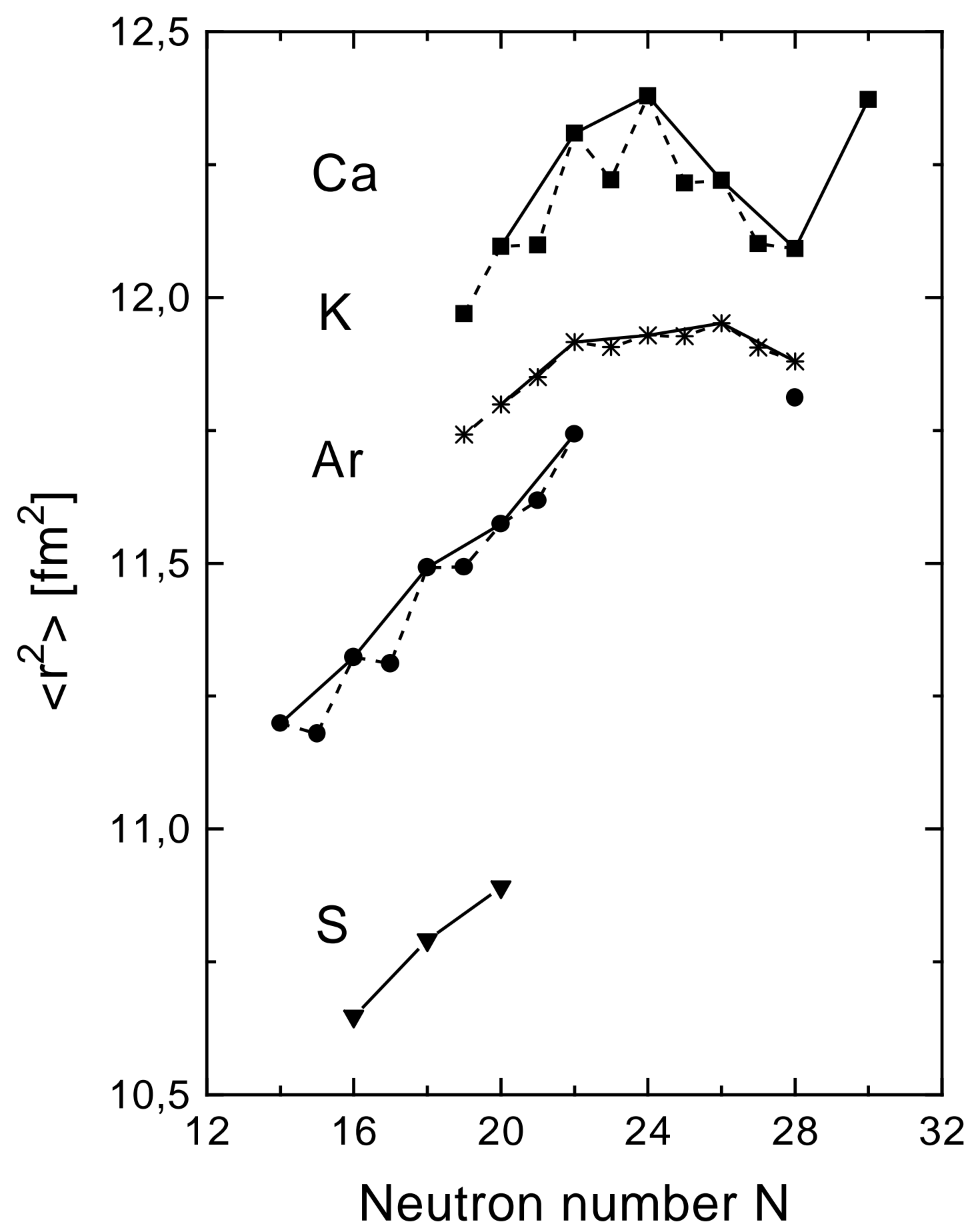

Figure 9: Mean square charge radii of calcium, potassium, argon and sulphur nuclei versus neutron number. Absolute radii are from ref. [2], differences within a sequence of isotopes have been evaluated from isotope shifts (refs. [4] for ${ }^{40-48} \mathrm{Ca}$, [13] for ${ }^{50} \mathrm{Ca}$, [12] for ${ }^{39} \mathrm{Ca}$, [48] from the data of [11] for ${ }^{38-47} \mathrm{~K}$, and this work for ${ }^{32-40} \mathrm{Ar}$ and ${ }^{46} \mathrm{Ar}$ ). 